\title{
The human immune system's response to carcinogenic and other infectious agents transmitted by mosquito vectors
}

\author{
Olle Johansson $^{1} \cdot$ Martin Ward $^{2}$ (D) \\ Received: 31 July 2016 / Accepted: 21 September 2016 / Published online: 27 October 2016 \\ (C) Springer-Verlag Berlin Heidelberg 2016
}

\begin{abstract}
It has been hypothesised that mosquitoes [Diptera: Culicidae] may play more of a role in certain cancers than is currently appreciated. Research links 33 infectious agents to cancer, 27 of which have a presence in mosquitoes, and that, in addition, mosquito saliva downregulates the immune system. The objective of this paper is to review the literature on the immune system and cancer-causing infectious agents, particularly those present in mosquitoes, with a view to establishing whether such infectious agents can, in the long run, defeat the immune system or be defeated by it. Many of the viruses, bacteria and parasites recognised by the International Agency for Research on Cancer (IARC) as carcinogenic and suspected by others as being involved in cancer have evolved numerous complex ways of avoiding, suppressing or altering the immune system's responses. These features, coupled with the multiplicity and variety of serious infectious agents carried by some species of mosquitoes and the adverse effects on the immune system of mosquito saliva, suggest that postmosquito bite the immune system is likely to be overwhelmed. In such a situation, immunisation strategies offer little chance of cancer prevention, unless a single or limited number of critical infectious agents can be isolated from the 'mosquito' cocktail. If that proves to be impossible cancer prevention will, therefore, if the hypothesis proves to be correct, rest on the twin strategies of environmentally controlling the mosquito population and humans avoiding being bitten. The latter
\end{abstract}

Martin Ward

martin@martinward200.plus.com

1 Department of Neuroscience, Karolinska Institute, 171

77 Stockholm, Sweden

2 The Pain Clinic, Bolsover S44 6BB, UK strategy will involve determining the factors that demark those being bitten from those that are not.

Keywords Mosquitoes $\cdot$ Health $\cdot$ Parasites $\cdot$ Infectious agents $\cdot$ Cancer

\section{Introduction}

It has been hypothesised that the mosquito may have more of a role in certain cancers than is currently appreciated (Ward et al. 2016; Benelli et al. 2016). In addition to the mosquito's known relationship with Burkett's lymphoma, 33 infectious agents have been linked with cancer and 27 of these have links, in one way or another, with mosquitoes (Ward et al. 2016). The authors couple this linkage with the known ability of mosquitoes to suppress the immune system. Hamster reticulum cell sarcoma can be transmitted through the bites of Aedes aegypti and there is an increased number of apoptotic cells in dengue-infected human monocyte cultures (Benelli et al. 2016).

Prostate cancer is one of the most common cancers in men, and the cause is largely unknown, although the generally accepted view is that it needs the hormone testosterone to grow. This latter contention is disputed on the basis that testosterone and its relation to prostate cancer is a myth, based on a blood test result from one patient in 1941 (Morgentaler 2006). This example of the confusion surrounding the cause and development of some cancers invites further consideration of whether mosquitoes have a connection with certain cancers. Of particular importance is the question of whether post a mosquito bite, the immune system can regain equilibrium, thus preventing possible cancer development, and if not, why not? 
There is a degree of support for the 'cocktail' hypothesis in that two common viruses, human papillomavirus (HPV) and Epstein-Barr virus (EBV), known to be associated with human cancers, are both present and may be collaborating with each other in most prostate cancer cases (Whitaker et al. 2012). Both viruses were present in more than half of the malignant cases with HPV alone present in about $70 \%$ of the malignant prostate cancers sampled.

Cancer viruses recognised by the IARC do not fall into a single viral class; they span the entire range of virology and include retroviruses, positive-stranded RNA viruses and both large and small double-stranded DNA viruses, (Moore and Chang 2010). The authors point out that immunity is an important factor as to whether cancer develops after contracting a potential cancer virus. To illustrate this they state that Kaposi's sarcoma herpes virus (KSHV) caused less than three Kaposi's sarcoma cases per year in the USA but the rate increased tens of thousands-fold among people with AIDS after immune suppression due to HIV. Many infectious agents linked with cancer have their own highly effective immune evasion strategies, which makes their control very difficult. Additionally, both viruses and bacteria have developed parallel strategies to neutralise host immunity, and as viral and bacterial diseases are frequently linked, they can take advantage of weaknesses created by another pathogen, (Finlay and McFadden 2006). There are a multitude of mechanisms both subtle and diverse by which viruses, bacteria, protozoa and various helminths evade the host's immune responses (Schmid-Hempel 2009).

Vector-borne diseases account for more than $17 \%$ of all infectious diseases, causing more than 1 million deaths annually and more than 2.5 billion people in over 100 countries are at risk of contracting dengue alone (WHO 2016). Understanding vector-borne diseases involves a study of many factors and the interactions between them, and we propose a six-part framework to aid that understanding.

1. Vector identification and classification. This includes understanding behavioural patterns, the biology and ecological requirements of vectors as well as correct vector classification, utilising modern methods such as DNA barcoding.

2. Vector-borne disease identification. This takes in both the types and range of diseases carried by vectors and which and how they are interchanged with intermediate hosts, as well as the factors causing mutations and variations in virulance.

3. Host identification. This involves identification of single, multiple and key vector hosts, host switching and the complex web of interactions between them.

4. Environmental changes. This involves understanding the effects on both vectors and hosts of environmental modifications such as dam construction, rapid urbanisation, use of nonbiodegradable packaging, temperature and rainfall fluctuations and changes in agricultural methods.
5. Control of vector-borne diseases. This requires an understanding of the innate immune response and, where relevant, when and how to utilise chemotherapy and immunisation.

6. Control of vectors. This ranges from predictive risk mapping of temperature data, to the use of simple solutions, such as limiting stagnant water, use of repellents and use of modern plant-borne mosquitocidals.

This paper is primarily concerned with the control of vector-borne diseases (no. 5 above), and in particular how the immune system responds to bites.

\section{The immune system and how infectious agents evade and counteract it}

This section is divided into four parts, part one briefly describes the immune system, part two how the immune system is affected by firstly, well-known mosquito-related infectious agents, and secondly cancerous and non-cancerous infectious agents, part three looks at specific immuneevasion strategies and part four lists general factors relevant to this part of the framework.

\section{The immune system}

The immune system is divided into two parts namely the innate and adaptive responses; the innate provides immediate host defence through neutrophils, monocytes, macrophages, complement, cytokines and acute phase proteins. The adaptive response has memory but response is not immediate, (Parkin and Cohen 2001). The immune system uses pathogen-recognition receptors (PRRs) to sense invading pathogens. PRRs recognise specific pathogen-associated molecular patterns (PAMPs) produced during the course of infection (Munoz-Jordan and Fredericksen 2010). The immune system responds to infectious agents by deploying interferons and specialised cells such as phagocytes. These specialised cells can internalise and destroy microbes and in addition recruit more immune cells (Finlay and McFadden 2006). Interferons (IFNs) are proteins (called cytokines) that inhibit virus replication and have pleiotropic effects on many aspects of cell physiology, including cell growth, cell motility and cell functions. Interferons consist of type I and type II, each act through different cell surface receptors and are structurally unrelated. There are many members of the type I superfamily but only one member of the type II family. An IFN-induced protein PKR13 has a pivotal role in host-virus interactions manifested by the fact that numerous viruses block its activation or action using a variety of biochemical strategies (Sen 2001). 


\section{Mosquito-related infectious agents}

Well known mosquito-related infections feature strongly in the immune-evasion literature; malaria has mechanisms for evading cytotoxic $\mathrm{T}$ cells and controlling protein export during liver infection to minimise immune recognition (Bertolino and Bowen (2015); Corradin and Levitskaya 2014; Montagna et al. 2014). It is also suggested that severe disease associated with dengue virus and West Nile virus (WNV) infections correlates with their ability to counteract the IFN- $\alpha / \beta$ response (Munoz-Jordan and Fredericksen 2010). Japanese encephalitis virus has mechanisms for coping with immune responses (Lin et al. 2004). Also reviewed have been the stress responses in flavivirus-infected cells (Blázquez et al. 2014). The flaviviruses cover dengue virus, yellow fever, WNV, Japanese encephalitis virus, Usutu and Zika viruses. These viruses can activate one or more of the three arms of the unfolded protein response (UPR). The neurovirulent WNV NY-99 upregulates all three pathways of the UPR. Interaction between the UPR and interferon signalling in flaviviral infections has been reported and there is evidence of connections with cellular metabolism, apoptosis and innate immunity. The authors, however, point to mixed observations and unclear mechanistic links. Neuroinvasive WNV infections are worse when there is impaired immune protection (James et al. 2016). A case study has been reported of a patient left with disabilities from WNV which became symptomatic again 11 years later following the effects arising from the installation of a new computer modem, (Johansson and Redmayne 2016).

\section{Cancerous and non-cancerous infectious agents}

Of the infectious agents identified with cancer, hepatitis $\mathrm{C}$ virus evades antiviral systems by modulating the function of innate immune proteins, including the signalling adaptor protein MAVS (mitochondrial antiviral-signalling protein) (Gokhale et al. 2014). Research shows how PAMPS trigger signalling, and that HCV, EBV, KSHV and Papillomaviridae have mechanisms for evading the immune responses (Navratil et al. 2010). Sen (2001) describes four parts of the IFN system, three of which are blocked by the Influenza virus, two parts are blocked respectively by the following: adenovirus, Epstein-Barr virus, hepatitis $\mathrm{B}$ and $\mathrm{C}$ viruses, human herpes virus 8 , human papilloma virus and one part by several other viruses including CMV. CMV infection results in significantly weaker responses to superinfection with influenza, human herpes virus or WNV (Cicin-Sain et al. 2012). All but influenza have been linked with cancer (Ward et al. 2016).

Of the infectious agents not currently linked with cancer, Visna and Langat viruses have evasion mechanisms and it is reported that poxviruses encode a unique set of secreted proteins that function as soluble cytokine receptors, or binding proteins, and modulate virus virulence (Alcami et al. 2000). The highly pathogenic H5N1 influenza viruses have a novel mechanism that counteracts the antiviral activity of interferons and TFN $-\alpha$ and the measles virus appears to use a related strategy (Seo et al. 2002). In addition, there is a suggestion that Echinococcus Granulosus antigen B exploits the activation of $\mathrm{T}$ helper cells by eliciting a non-protective $\mathrm{Th} 2$ cell response (Rigano et al. 2001).

\section{Evasion strategies utilised by infectious agents}

Specific strategies that viruses and bacteria use to avoid immune recognition, response and destruction, have been identified (Finlay and McFadden 2006; Schmid-Hempel 2009). These strategies are both complex and diverse, as illustrated below.

Finlay and McFadden (2006) state that a pathogen can avoid, alter or mimic host immune modulators, for example, a virus can seek to change its external surface by studding it with potentially immunomodulatory viral proteins. Bacteria, for example, Streptococcus pneumonia, can mask their surfaces by expressing a carbohydrate capsule, to prevent antibody and complement deposition on its surface, thus avoiding phagocytic clearance. Haemophilus influenzae, Escherichia coli $\mathrm{K} 1$ and Neisseria meningitidis operate similar strategies. Gram-negative bacteria have developed secretion systems, called effectors, which can kill host cells, and reprogram vesicular transport to enhance intracellular parasitism: both Streptococcus pyogenes and Mycobacterium tuberculosis utilise this strategy.

Salmonella, Porphyromonas gingivalis and Listeria have developed strategies to alter their surface molecules so that they are less recognisable. Neisseria species vary immunodominant molecules (named antigenic variation) so that they appear different to what the immune system has detected previously. Hepatitis C, HIV and CMV can both evade the immune response and confound attempts at producing vaccines.

The innate response is to send out phagocytes to digest and kill microbes but Yersinia species, Shigella, Listeria moncytogenes, Rickettsia, Legionella pneumophila and Salmonella species have developed strategies to neutralise phagocytic activity. M. tuberculosis has many surface glycolipids and carbohydrates that prevent phagosome acidification and alter phagosomes. Many viruses have evolved protective mechanisms to counter the antimicrobial functions of nitric acid and reactivate oxygen radicals generated by activated phagocytes. There is evidence that toll-like receptors (TLRs) transduce the earliest signals of the innate immune responses to microbial infections and that anti-TLR strategies are likely common amongst all successful pathogens. Poxviruses and hepatitis $\mathrm{A}$ and $\mathrm{C}$ can manipulate signalling by TLRs.

Pathogens such as Salmonella have developed strategies that alter their surface structure to decrease insertion of 
peptides and resulting lysis, they can encode transport systems that remove the peptides, and they can secrete proteases that degrade these peptides. HIV, HTLV-1 and Vaccinia incorporate host inhibitors into the virus envelope. Staphylococcus aureus and Shigella have a strategy of expressing anti-cytokine proteins that can modulate pathway regulators that influence the stability, trafficking or signalling of infected cell receptors.

Herpes viruses can cause dysfunction of the NK cells that contribute to the development of acquired cellular immune responses and Helicobacter pylori, for example, interferes directly with acquired immunity by blocking type $1 \mathrm{~T}$ helper (Th1) cells development and producing a vacuolating toxin, $\mathrm{VacH}$, which blocks $\mathrm{T}$ cell proliferation. Chlamydia and Salmonella enterica have strategies for either accelerating or inhibiting the cell death pathways of the infected cell.

Schmid-Hempel (2009) states that parasites can conceal themselves by invading immune-privileged tissue, such as the central nervous system or the eye. Some parasitoids place their eggs inside fat tissue that is not well patrolled by the host's immune system. Other parasites store surface variants that are successively expressed; for example, Plasmodium falciparum has approximately 60 stored variants and Trypanosoma brucei has several hundred. Evasion can also involve mutants, with HIV mutants arising with sufficient frequency to outpace the immune system. Plasmodium, schistosomes and nematodes have been reported to produce competing ligands to impede recognition by the host, and Toxoplasma modifies its host vacuole membrane with its own proteins to prevent further immune response. Myxoma virus, adenovirus, and Vaccinia virus interfere with host cell apoptosis, and Legionella, Coxiella and Chlamydia interfere with vacuole maturation in their host cells.

Pathogenesis can lead to a host's resources being depleted, destroy tissue and adversely affect behaviour.

Parasite-released toxins, produced by, for example, Clostridia, Bacillus anthracis, and human rhinovirus, interfere with the IFN response, allowing host invasion and spread. In some cases, virulence emerges because the pathogen enters a body part that seemingly has nothing to do with transmission. Finally, bacteria may need to extract host resources for its survival for example extracting iron, and some parasites even manage to feed on the host's immune response; for example, Leishmania grows by consuming cytokines.

\section{General factors}

In both humans and birds, there is individual variation in pathogen susceptibility due to underlying variations at different parts of the immune system; individuals are affected differently once they become infected, and innate genes are constantly interacting and evolving with a changing pathogen flora (Hellgren 2015). Within tumours, the IARC-recognised viruses are generally latent, allowing them to hide from the immune system. The Herpes virus family, in particular EBV and KSHV, has latent viral tissue culture systems; during latency, the viral genome replicates in tandem with the host cell using the replication machinery of the cell (Moore and Chang 2010). There is also the concept of 'hit-and-run' viruses in which the viral genes are lost as the tumour begins to mature (Moore and Chang 2010; Shen et al. 1997). M. tuberculosis is taken up by macrophages but prevents the fusion of the phagosome with the lysosome, protecting itself from the bactericidal actions of the lysosomal contents and Listeria, Toxoplasma gondii, Borrelia burgdorferi and Treponema pallidum can avoid elimination by antibodies (Janeway et al. 2001). Parasites have the ability to successfully infect a wide range of host species of broad ancestry (Hellgren et al. 2009; Webster et al. 2016). Additionally, avian malaria parasites have developed evolutionary independence (Hellgren et al. 2015). Thus, mosquitoes taking a blood meal from different hosts are exposed to a variety of parasites. Polyomaviruses, which subclinically may infect the general population, may become significant diseases in patients with impaired immune functions (Allander et al. 2007; Babakir-Mina et al.; 2011; Abedi Kiasari et al. 2011; Dalianis and Hirsch 2013).

\section{The mosquito's influence on the immune system}

The mosquito can suppress the human immune system through its saliva and transmission of infectious agents. Mosquito saliva has the capacity to activate dermal mast cells and to induce local inflammatory cell influx (Depinay et al. 2006). Mosquito bites consistently induced macrophage inflammatory protein 2 (MIP-2) in the skin and interleukin10 (IL-10) in draining lymph nodes and downregulates antigen-specific $\mathrm{T}$ cell responses by a mechanism dependent on mast cells and mediated by IL-10. Plasmodium berghei sporozoites delivered into mice through mosquito bites were more infectious than when sporozoites were injected intravenously, suggesting a facilitating role of saliva for parasite transmission. Compared with arbovirus infection initiated in the absence of the mosquito or its saliva, infection via mosquito saliva leads to an increase in virus transmission, host susceptibility, viraemia, disease progression and mortality (Schneider and Higgs 2008). Mice infected with WNV through the bite of a single infected Culex tarsalis mosquito exhibited five- to tenfold higher viraemia and tissue titers at 24 and $48 \mathrm{~h}$ post inoculation and faster neuroinvasion than mice given a median mosquito-inoculated dose of WNV by needle (Styer et al. 2011). In addition, inoculation of mice with WNV mixed with salivary gland extract led to higher viremia, demonstrating that mosquito saliva is the major cause of mosquito-induced enhancement. 
It has been stated that in dengue virus, serine protease activity in Ae. aegypti saliva augmented virus infectivity and that mosquito saliva contains a potent mixture of secreted molecules that can affect vascular constriction, blood coagulation, platelet aggregation, inflammation, immunity and angiogenesis (Conway et al. 2014). It has been found that the host immune response to mosquito-transmitted chikungunya virus differs from that elicited by needleinoculated virus (Thangamani et al. 2010). Compared to expression with the Sinbis virus alone, IL-10 expression showed a 7.6 -fold increase by $72 \mathrm{~h}$ post inoculation in mice receiving mosquito salivary gland extract with the virus (Schneider et al. 2004). Differences in saliva contents between species may affect pathogen transmission and successful viruses co-evolve with mosquito vectors (Colpitts et al. 2012).

There are several types of viral pathogens that cause disease in mosquitoes. Two of the most common are the baculoviruses and cytoplasmic polyhedrosis viruses (Reoviridae; Cypovirus). Other major types of viruses are the densoviruses and the iridoviruses (Becnel and White 2007).

Mosquitoes are well known for their ability to transmit deadly and debilitating pathogens and parasites, such as filariasis, malaria, WNV, chikungunya and increasingly dengue and Zika viruses (Benelli and Melhorn 2016). A comprehensive list of the main pathogens and parasites associated with mosquito vectors is available (see Murugan et al. 2016). There are many less well known pathogens carried by mosquitoes, for example 83 bacterial species belonging to 31 bacterial genera in the midgut of Culex quinquefasciatus mosquitoes (Chandel et al. 2013). All the species belong to three phyla, Proteobacteria, Firmicutes and Actinobacteria. The diversity of microbes was quite high in all of their samples. They state that a fraction of mosquito midgut inhabitants could be common for different mosquito species.

Recently reported facts about mosquitoes both add to the complexity of immune evasion and may have implications for cancer prevention, if the connection between the two can be proven. It has been reported that WNV was isolated from male mosquitoes, suggesting that mosquitoes become infected by means other than by blood feeding, possibly by transovarial transmission (Anderson et al. 2006). There are also reports of B. burgdorferi (the cause of Lyme disease) in Culex mosquitoes, (Anderson et al. 2006; Zákovská et al. 2002) and in Germany DNA of Borrelia afzelii, Borrelia bavariensis and Borrelia garinii has been detected in four distinct genera (Aedes, Culiseta, Culex and Ochlerotatus), with positive samples found in adult specimens from wild-caught larvae, suggesting transstadial and/or transovarial transmission (Melaun et al. 2016). This adds additional complexity to the understanding of how a variety of host species contribute to transmission and human infection (Webster et al. 2016). A biofilm in Lyme's disease has been discovered that boosts therapy resistance by nearly 1000 times (Sapi et al. 2016). It has also been observed that the dengue structure differs at the temperatures of its human and mosquito hosts (Zhang et al. 2013).

The next section discusses the interactions of the factors set out above, provides an alternative to the 'hit-and-run' hypothesis and considers the implications arising from male and female mosquitoes carrying similar, possibly endogenous, diseases.

\section{The links between mosquitoes and pathogens}

The literature shows that viruses, bacteria and parasites have developed numerous and complex ways of evading the immune system and protecting themselves against host immune responses. Many of the pathogens identified in the previous section have been linked with cancer and many have been linked with mosquitoes. All those linked with mosquitoes benefit from the ability of mosquito saliva to suppress the immune system, increase viraemia and inflammation. Table 1 summarises the position.

Cancer tumour viruses carried by mosquitoes and injected into humans have a greater chance of causing mortality or serious illness than viruses picked up by other means. It has been recorded that one person can receive a thousand infectious bites per annum (Ferguson et al. 2010). Recent findings that some mosquito species can carry Borrelia illustrate how much more we need to understand about mosquitoes.

Tumours are known to contain numerous infectious agents, the question is how did they get there, and what is their relationship to cancer development? The present assumption is that the immunosuppressed, brought about through HIV, after transplants or developing cancer are then prone to catching infectious agents in a linear fashion. However, in most developed countries, water, food and hygiene standards are such that encountering these infectious agents in the variety present in tumours should be minimal. Encountering them from a mosquito bite seems much more likely. Various studies suggest that the effective dose for successful infection may often be surprisingly small and that co-operatively acting bacteria tend to have higher infective doses than bacteria where the factors are injected individually (Schmid-Hempel 2009).

Will the immune system's response to a mosquito bite be to ultimately regain control? If the mosquito cocktail contains several cancer-related infectious agents, the answer may be no, but it may be yes if some mosquito species cannot transmit cancerous agents and/or some people are better adapted than others to deal with particular infections (Hellgren 2015). Other exogenous factors may influence developments; for example, it has been speculated that disturbance of the immune system by electromagnetic fields is a potentially underlying cause for cellular damage and tissue repair reduction (Johansson 2009). 
Table 1 Infectious agents with immune evasion strategies, their links with cancer and mosquitoes

\begin{tabular}{llll}
\hline $\begin{array}{l}\text { Infectious agent with } \\
\text { evasion capability }\end{array}$ & $\begin{array}{l}\text { Genus/author } \\
\text { (where known). }\end{array}$ & $\begin{array}{l}\text { Linked } \\
\text { with }\end{array}$ & $\begin{array}{l}\text { Linked } \\
\text { with }\end{array}$ \\
$\begin{array}{l}\text { (species if not the } \\
\text { same) }\end{array}$ & & cancers & mosquitoes \\
\hline
\end{tabular}

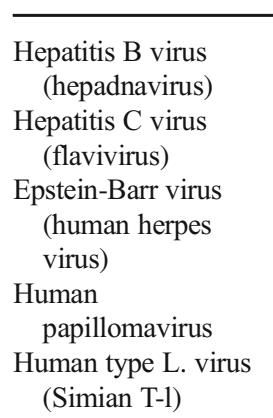

Karposi's sarcoma virus

Merkel cell polyomavirus

Helicobacter pylori

Adenovirus

Orthohepadnavirus/
Blumb 1996
Hepacivirus/Houghton
1989
Lymphocryptovirus/
Epstein-Barr1964

Yes Yes

Alpha, Beta, Gamma,

Mupa, Nupa

Deltaretrovirus/Rous Yes No 1935

Human herpes virus 8/Mkoposi 1872

Orthopolyomavirus

Yes Yes

Helicobacter/Goodwin

Yes

No et al. 1989

Adenoviridae/Rowe et al. Yes 1953

Cytomegaloma virus

Malaria Plasmodium falciparum

Streptococcus pyogenes

Chlamydia

Staphlococcus aureus

Staphlococcus epidermatis

Helminths (flatworms and roundworms)

West Nile virus

Japanese encephalitis virus

Schistosomes

Herpes

Mycobacteria tuberculosis

Salmonella typhus (enterica)

Blastomyces dermatitis

Yersina pestis

Listeria monocytogene

Legionella pneumophila

Coxiella burnetii

Toxoplasma gondii

Vaccinia

Clostridia

Bacillus anthracis
Cytomegalomavirus Yes Yes

Plasmodium Yes Yes

Streptococcus/Lancefield Yes Yes 1928

Chlamydia/Busacco 1935 Yes No

Staphylococcus/ Yes Yes

Rosenbach 1889

Staphylococcus/Winslow No Yes and Winslow 1908

Flavivirus

Flavivirus

Schistosoma

Herpesviridae

Mycobacteria/Koch 1882 Yes

Salmonella/Lignieres Yes 1990

Blastomyces/Gilchrist and Stokes 1898

Yersinia/Lehmann and Neuman 1896

Listeria/Pirie 1940

Legionella/Brenner et al. No 1979

Coxiella/Derrick 1939 No No

Toxoplasma Nicolle \& No $\quad$ Yes Marceaux 1909

Orthopoxvirus

Clostridium/Prazmowski 1880

Bacillus/Cohn 1872
Table 1 (continued)

Infectious agent with evasion capability (species if not the Linked Linked with with cancers mosquitoes same)

\begin{tabular}{|c|c|c|c|}
\hline $\begin{array}{c}\text { Haemophilus } \\
\text { influenzae }\end{array}$ & $\begin{array}{l}\text { Haemophilus/Lehmann } \\
\text { and Newman } 1896\end{array}$ & No & No \\
\hline Escherichia coli $\mathrm{K} 1$ & Escherichia/Migula 1895 & No & Yes \\
\hline $\begin{array}{l}\text { Neisseria } \\
\quad \text { meningitidis }\end{array}$ & $\begin{array}{l}\text { Neisseria/Albrecht and } \\
\text { Ghon1901 }\end{array}$ & No & No \\
\hline Trypanosoma brucei & $\begin{array}{l}\text { Trypanosoma/Plimmer } \\
\text { and Bradford } 1899\end{array}$ & No & No \\
\hline Leishmania & Leishmania/Ross 1903 & No & No \\
\hline $\begin{array}{l}\text { Porphyomonas } \\
\text { gingivalis }\end{array}$ & $\begin{array}{l}\text { Porphyomonas/ } \\
\text { Coykendall et al. } 1980\end{array}$ & No & No \\
\hline Rickettsiae & $\begin{array}{l}\text { Rickettsia/da Rocha-Lima } \\
\quad 1916\end{array}$ & No & No \\
\hline Shigella dysenteriae & $\begin{array}{l}\text { Shigella/Castellani and } \\
\text { Chalmers } 1919\end{array}$ & No & No \\
\hline $\begin{array}{r}\text { Influenza A virus } \\
\text { subtype H5N1 }\end{array}$ & Influenza virus A & No & No \\
\hline Langat virus & Flavivirus & No & No \\
\hline Human rhinovirus & Enterovirus & No & No \\
\hline $\begin{array}{c}\text { Echinococcus } \\
\text { granulosis }\end{array}$ & $\begin{array}{l}\text { Echinococcus/Batsch } \\
\quad 1786\end{array}$ & No & No \\
\hline Myxoma virus & Leporipoxvirus & No & No \\
\hline Treponema pallidum & $\begin{array}{l}\text { Treponema/Schaudinn } \\
\text { and Hoffman } 1905\end{array}$ & No & No \\
\hline
\end{tabular}

The finding that male mosquitoes carry similar infectious agents to the female and that pathogens have been discovered in mosquitoes from wild-caught larvae suggests that mosquitoes have endogenous infections. If the endogenous infections are both uniform and potentially cancer-causing, then reliance on intermediate hosts may be less important than supposed.

The 'hit-and-run' theory seeks to explain why viruses do not develop within tumours. To a layman, hit-and-run will most likely conjure up an image of a car crash in which the causal driver runs away from the scene. However, in this scenario the damage done is usually both instant and complete, with no progressive subsequent damage. We propose a more prosaic explanation for tumour development, called the 'bake-a-cake' phenomenon. The mosquito provides numerous ingredients (viruses, parasites and bacteria) together with a mixing agent (its saliva). Subsequent to a bite, inflammatory processes take place equivalent to a slow baking of the mixed ingredients. When a cake is fully baked the ingredients, for example, dried fruit and nuts, do not increase in number and mixing agents like alcohol can disappear. Thus, with a tumour, the initial pathogens do not increase in number, and the mixing agent, saliva disappears. Just two ingredients can work synergistically to produce different effects, (HCMV) IE1 and IE2 gene products co-operate with adenovirus E1A gene to transform primary baby rat kidney cells, and the IE1 and IE2 are present only transiently (Shen et al. 1997). 
What, therefore, are the implications for cancer prevention? In the absence of any initial febrile responses to mosquito bites, the medical profession may not connect a presenting ailment with a bite. Patients may be unaware that they have been bitten and, even if aware, the bites from some species of mosquito are not presently regarded as dangerous and it is assumed that the immune system will restore equilibrium. As a consequence, until the mosquito-cancer connection is rigorously tested, tests for identifying cancer will remain as they are now. Because many pathogens have highly effective mechanisms to subvert the human immune system, developing vaccines and controlling these pathogens, even if detected, is extremely difficult (Finlay and McFadden 2006). It has been pointed out that as viruses in tumours are generally latent, antiviral drugs targeting the viral replication machinery are ineffective in treating mature tumours (Moore and Chang 2010). It has been reported that the most advanced tetravalent live-attenuated dengue fever vaccine candidate showed a poor $30 \%$ overall efficacy rate in a recently published phase 2 clinical trial (Zhang et al. 2013). Nevertheless, there is a proposal to use a combination approach in cancer immunotherapy that includes dengue vaccination (Lyday et al. 2015). If the mosquito cancer-cocktail hypothesis is proven then using dengue in this way may be more problematic than they suppose as they state that secondary infections carry greater risks. The use of green synthesised nanoparticles has interesting potential but requires further investigation regarding their environmental impact (Benelli 2015).

In view of this complexity, prevention strategies for any mosquito-caused cancers would, therefore, have to focus on bite avoidance, through repellents or control. This is not an easy task either, as illustated by several comprehensive reviews of the biological complexities involved in environmental mosquito control (Ferguson et al. 2010; Benelli and Melhorn 2016; Webster et al. 2016). The authors conclude that mosquitoes need to be controlled at multiple points in the continuum from egg to adult. It is doubtful that all individuals will be able or willing to take steps to avoid being bitten, as this may involve continuous use of repellent, removal of garden water features, and maybe uprooting trees, bushes and flowers known to harbour or provide food for mosquitoes.

\section{Conclusions}

Only a small proportion of people infected with infectious agents associated with cancer go on to develop tumours. However, cancers that are related to immunosuppression are candidates for being caused by tumour viruses and the effective dose for successful infection can be small. Mosquito saliva causes immunosuppression and mosquitoes can carry a multiplicity of infectious agents, some known to be carcinogenic, and many of which have different strategies to evade a strong immune system let alone one already weakened by the contents of saliva. Therefore, subsequent to a bite, the immune system will likely be overwhelmed. Whether it can regain equilibrium may well depend on how many carcinogenic infectious agents are transmitted, either from one or multiple bites and individual immune system characteristics.

Cancers identified in the early stages may allow antiviral drugs to prevent mature tumours from developing but not when they are mature. If a mosquito-cancer connection can be established then earlier identification of cancer may be possible. Vaccinations against some infectious agents are also problematic. Mosquito control strategies have numerous problems to overcome, and bite avoidance relies on protective measures that many humans will currently find difficult to follow, unless a unique characteristic like a repellent hormone can be found and utilised in a simple and cost-effective way. What is required is further research across all six parts of the framework in order to obtain a richer understanding of all species of human-biting mosquitoes.

Acknowledgments We would like to thank Prof. Giovanni Benelli for his helpful comments, which have improved an earlier version of the manuscript.

\section{Compliance with ethical standards}

Conflict of interest The authors declare that they have no conflicts of interest.

Funding None declared.

\section{References}

Abedi Kiasari B, Vallely PJ, Klapper PE (2011) Merkel cell polyomavirus DNA in immunocompetent and immunocompromised patients with respiratory disease. J Med Virol Dec 83(12):2220-2224

Alcami A, Symons JA, Smith GL (2000) The vaccinia virus soluble alpha/beta interferon (IFN) receptor binds to the cell surface and protects cells from the anti-viral effects of IFN. J Virol 74(23): $11230-11239$

Allander T, Andreasson K, Gupta S, Bjerkner A, Bogdanovic G, Persson MA et al (2007) Identification of a third human polyomavirus. J Virol 81(8):4130-4136

Anderson JF, Andreadis TG, Main AJ, Ferrandino FJ, Vossbrink CR (2006) West Nile virus from female and male mosquitoes (Diptera: Culicidae) in subterranean, ground, and canopy habitats in Connecticut. J Med Entomol 43(5):1010-1019

Babakir-Mina M, Ciccozzi M, Perno CF, Ciotti M (2011) The novel KI, WU, MC polyomaviruses: possible human pathogens? New Microbiol 34(1):1-8

Becnel JJ, White SE (2007) Mosquito pathogenic viruses - the last 20 years. J Am Mosq Control Assoc 23:36-49

Benelli G (2015) Research in mosquito control: current challenges for a brighter future. Parasitol Res 114:2801-2805. doi:10.1007 /s00436-015-4586-9 
Benelli G, Melhorn H (2016) Declining malaria, rising of dengue and Zika virus: insights for mosquito vector control. Parasitol Res 115: 1747-1754. doi:10.1007/s00436-016-4971-z

Benelli G, Lo Iacono A, Canale A, Mehlhorn H (2016) Mosquito vectors and the spread of cancer: an overlooked connection? Parasitol Res 115(6):2131-2137

Bertolino P, Bowen DG (2015) Malaria and the liver: immunolological hide-and-seek or subversion of immunity from within. Front Microbiol 6:41

Blázquez A-B, Escribo-Romero E, Merino-Ramos T, Saiz J-C, MartinAcerbes M (2014) Stress responses in flavivirus-infected cells: activation of unfolded protein response and autophagy. Front Microbiol 5:266

Chandel K, Mendki MI, Parikh RY, Kulkarni G, Tikar SN, Sukumaran D et al (2013) Midgut microbial community of Culex quinquefasciatus mosquito populations from India. PloS one 8(11):e80453

Cicin-Sain L., Brien J.D., Uhrlaub J. L., Drabig A., Marandu T. F., Nikolich-Zugich J., (2012). Cytomegaloma virus infection impairs immune responses and accentuates T-cell pool changes observed in mice with aging. PLOS pathogens. doi:10.1371/journal. ppat.1002849.

Colpitts TM, Conway MJ, Montgomery RR, Fikrig E (2012) West Nile virus: biology, transmission, and human infection. Clin MicrobiolRev 25(4):635-648

Conway MJ, Watson AM, Colpitts TM, Dragovic SM, Zhiyong L, Wang P et al (2014) Mosquito saliva serine protease enhances dissemination of dengue virus into the mammalian host. J Virol 88(1):164-175

Corradin G, Levitskaya J (2014) Priming of CD8(+) T-cell responses to liver stage malaria parasite antigens. Front Immunol 5:5.527

Dalianis T, Hirsch HH (2013) Human polyomaviruses in disease and cancer. Virology 437(2):63-72

Depinay N, Hacini F, Begdadi W, Peronet R, Mecheri S (2006) Mast cell dependent down regulation of antigen-specific immune responses by mosquito bites. J Immunol 176:4141-4146

Ferguson HM, Dornhaus A, Beeche A, Borgemeister C, Gottlieb M, Mulia MS et al (2010) Ecology: a prerequisite for malaria elimination and eradication. doi:10.1371/journal.pmed.1000303

Finlay BB, McFadden G (2006) Anti-immunology: evasion of the host immune system by bacterial and viral pathogens. Cell 124:767-782

Gokhale NS, Vazquez C, Horner SM (2014) Hepatitis C virus: strategies to evade antiviral responses. Futur Virol 9(12):1061-1075

Hellgren O (2015) Allelic variation at innate immune genes (avian $\beta$ defensins) within a natural population of great tits. J Avian Biol 46: $113-119$

Hellgren O, Perez-Tris J, Bensch S (2009) A jack-of-all-trades and still a master of some: prevalence and host range in avian malaria and related blood parasites. Ecology 90:2840-2849

Hellgren O, Atkinson CT, Bensch S, Albayrak T, Dimitrov D, Ewen JG et al (2015) Global phylogeography of the avian malaria pathogen Plasmodium relictum based on MSP1 allelic diversity. Ecography 8(8):842

James EA, Gates TJ, Lafond RE, Yamamoto S, Ni C, Mai D et al (2016) Neuroinvasive West Nile infection elicits elevated and atypically polarized T-cell responses that promote a pathogenic outcome. PLoS Pathog. doi:10.1371/journal.ppat.1005375

Janeway C, Jr A, Travers P, Walport M et al (2001) Pathogens have evolved various means of evading or subverting normal host defences. Immunobiology: the immune system in health and disease, 5th edn. Garland Science, New York, Http://www.ncbi. nlm.nih.gov/books/NBK27176

Johansson O (2009) Disturbance of the immune system by electromagnetic fields - a potentially underlying cause for cellular damage and tissue repair reduction which could lead to disease and impairment. Pathophysiology 16:157-177
Johansson O, Redmayne (2016) Exacerbation of demylinating syndrome after exposure to wireless modem with public hotspot. Electromagn Biol Med. doi:10.3109/15368378.2015.1107839

Lin R-J, Liao C-L, Lin E, Lin Y-L (2004) Blocking of the alpha- interferon induced Jak-stat signalling pathway by Japanese encephalitis virus infection. J Virol 78(17):9285-9294

Lyday B, Chen T, Kesari S, Minev B (2015) Overcoming tumor immune evasion with an unique arbovirus. J Trans Med 13:3

Melaun C, Zotzmann S, Santaella VG, Werblow A, ZumkowskiXylander H, Kraiczy P et al (2016) Occurrence of Borrelia burgdorferi s.l. in different genera of mosquitoes (Culicidae) in Central Europe. Ticks Tick Borne Dis 7(2):256-263

Montagna GN, Beigier-Bompadre M, Becker M, Kroczek RA, Kaufmann SH, Matuschewski K (2014) Antigen export during liver infection of the malaria parasite augments protective immunity. M Bio 29:5(4)

Moore PS, Chang Y (2010) Why do viruses cause cancer? Highlights of the first century of human tumour virology. Nate Rev Cancer 10: 878-889

Morgentaler A (2006) Testosterone and prostate cancer: an historical perspective on a modern myth. Eur Urol 50:935-939

Munoz-Jordan JL, Fredericksen BL (2010) How flaviviruses activate and suppress the interferon response. Viruses 2(2):676-691

Murugan K, Vadivalagan C, Karthika P, Panneerselvam C, Paulpandi M, Subramaniam J et al (2016) DNA barcoding and molecular evolution of mosquito vectors of medical and veterinary importance. Parasitol Res 115:107-121. doi:10.1007/s00436-015-4726-2

Navratil V, de Chassey B, Meyniel L, Pradezynski F, Andre P, RabourdinCombe $C$ et al (2010) System-level comparison of protein-protein interactions between viruses and the human type 1 interferon system network. J Proteome Res 9:3527-3536

Parkin J, Cohen B (2001) An overview of the immune system. The Lancet 357(9270):1777-1789

Rigano R, Profumo E, Brushi F, Carulli G, Azzara A, Ioppolo S et al (2001) Modulation of human immune response by Echinococcus granulosus antigen $\mathrm{B}$ and its possible role in evading host defences. Infect Immmun 69(1):288-296

Sapi E, Balasubramanian K, Poruri A, Maghsoudlou JS, Socarras KM, Timmarajou AV (2016) Evidence of in vivo existence of Borrelia biofilm in borrelial lymphocytomas. Eur J Microbiol Immunol. doi:10.1556/1886.2015.00049

Schmid-Hempel P (2009) Immune defence, parasite evasion strategies and their relevance for 'macroscopic phenomena' such as virulence. Philos Trans R Soc Lond B Biol Sci 364(1513):85-98

Schneider BS, Higgs S (2008) The enhancement of arbovirus transmission and disease by mosquito saliva is associated with modulation of the host immune response. Trans $\mathrm{R}$ Soc Trop Med Hyg 102(5):600-608

Schneider BS, Soong L, Zeider NS, Higgs S (2004) Aedes aegypti salivary gland extracts modulate anti-viral and $\mathrm{TH} 1 / \mathrm{TH} 2$ cytokine responses to Sindbis virus infection. Viral Immun 17(4):565-573

Sen GC (2001) Viruses and interferons. Annu Rev Microbiol 55:255-281

Seo SH, Hoffman E, Webster RG (2002) Lethal H5N1 influenza virus escape host anti-viral cytokine responses. Nat Med 8(9):950-954

Shen Y, Zhu H, Shenk T (1997) Human cytomegalomavirus IE1 and IE2 proteins are mutagenic and mediate "hit-and-run" oncogenic transformation in cooperation with the adenovirus E1A proteins. Proc Natl Acad Sci 94(7):3341-3345

Styer M, Lim P-Y, Louie KL, Albright RG, Kramer LD, Bernard KA (2011) Mosquito saliva causes enhancement of West Nile virus infection in mice. J Virol 85(4):1517-1527

Thangamani S, Higgs S, Zieglers S, Vanlandingham D, Tesh R, Wikel S (2010) Host immune response to mosquito-transmitted Chikungunya virus differs from that elicited by needle inoculated virus. PLoS One 5(8):e12137 
Ward M, Ward A, Johansson O (2016) Does the mosquito have more of a role in certain cancers than is currently appreciated? The mosquito cocktail hypothesis. Medical Hypothesis 86:85-91

Webster JP, Gower CM, Knowles SCL, Molyneux DH, Fenton A (2016) One health-an ecological and evolutionary framework for tackling Neglected Zoonotic Diseases. doi:10.1111/eva.12341.

Whitaker NJ, Glenn WK, Sahrudin A, Orde MM, Delprado W, Lawson JS (2012) Human papillomavirus and Epstein Barr virus in prostate cancer: koilocytes indicate potential oncogenic influences of human papillomavirus in prostate cancer. Prostate. doi:10.1002/pros.22562
WHO (2016) Vector-borne diseases. Media centre factsheet

Zákovská A, Nejedia P, Holiková A, Dendis M (2002) Positive findings of Borrelia burgdoreri in Culex (Culex) pipiens pipiens larvae in the surroundings of Brno city determined by the PCR method. Ann Agric Environ Med 9(2):257-259

Zhang X, Sheng J, Plevka P, Kuhn RJ, Diamond MS, Rossman MG (2013) Dengue structure differs at the temperatures of its human and mosquito hosts. Proc Natl Acad Sci 110(17):6795-6799 